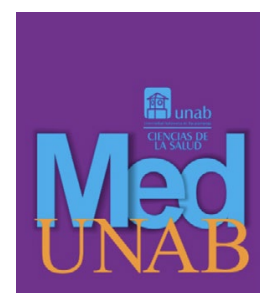

REVISTA DE LA FACULTAD

DE CIENCIAS DE LA SALUD

\title{
Melanoma metastásico a vejiga
}

\section{Metastatic melanoma to the bladder}

Melanoma metastático na bexiga

\section{Ana María Ortiz-Zableh, MD. ${ }^{1}$ (D), Juan Manuel Sandoval-Ojeda, MD. ${ }^{1}$ (D), Natalia Patiño-Covelli, MD. ${ }^{2}$ (D), Isabel Cristina Bolívar-Aguilera, MD., Esp. ${ }^{3}$ (D), Alfredo Ortiz-Azuero, MD., Esp. ${ }^{4}$ (D)}

1. Médico, Residente de Urología. Universidad Autónoma de Bucaramanga. Bucaramanga, Santander, Colombia.

2. Médico. Universidad Autónoma de Bucaramanga. Bucaramanga, Santander, Colombia.

3. Médico, Especialista en Patología. Clínica FOSCAL. Bucaramanga, Santander, Colombia.

4. Médico, Especialista en Urología, Docente postgrado Urología. Centro Urológico FOSCAL, Universidad Autónoma de Bucaramanga. Bucaramanga, Santander, Colombia.

Correspondencia. Ana María Ortiz Zableh. Universidad Autónoma de Bucaramanga, Campus el Bosque. Floridablanca, Santander, Colombia. Email. aortiz779@unab.edu.co

\section{INFORMACIÓN DEL ARTÍCULO:}

Artículo recibido: 21 de mayo de 2020

Artículo aceptado: 21 de octubre de 2021

DOI: https://doi.org/10.29375/01237047.3912

Cómo citar. Ortiz-Zableh AM, Sandoval-Ojeda JM, Patiño-Covelli N, Bolívar-Aguilera I, OrtizAzuero A. Melanoma metastásico a vejiga. MedUNAB [Internet]. 2021;24(3):353-358. doi: https://doi. org/10.29375/01237047.3912

\section{RESUMEN}

Introducción. El compromiso tumoral metastásico del melanoma al tracto genitourinario es frecuente, pero, la metástasis a vejiga es rara, constituye menos del $2 \%$ de los casos. Sin embargo, en autopsias realizadas a pacientes con melanoma se ha encontrado metástasis en la vejiga en entre un $18 \%$ y un $37 \%$ de los casos, lo que la convierte en la segunda en incidencia posterior al adenocarcinoma gástrico. La media de supervivencia suele ser entre 6 - 7.5 meses. El objetivo de este trabajo es presentar el caso de un melanoma metastásico a vejiga, entidad poco frecuente y poco diagnosticada por ser la mayoría de las veces asintomática. Presentación del caso. Paciente femenina de 62 años, con antecedente de melanoma al nivel del primer artejo del pie, con manejo quirúrgico y farmacológico. Consultó por hematuria. La cistoscopia evidenció una lesión única sólida, eritematosa, con necrosis y fácil sangrado y se indicó realizar resección transuretral (RTU). La patología demostró compromiso por melanoma ulcerado metastásico. Se inició manejo de segunda línea (Pembrolizumab) y presentó progresión a miembros superiores y recaída a nivel vesical. La paciente falleció un año después. Discusión. Las metástasis de melanoma al tracto genitourinario son frecuentes, pero las metástasis vesicales aisladas 
son raras. El tratamiento suele ser RTU de la lesión, cistectomía, quimioterapia y radioterapia. La RTU es curativa para las lesiones restringidas al epitelio, aunque la cistectomía radical suele ser la terapia de elección ante un paciente con un tumor localizado. El Pembrolizumab ha demostrado aumentar la supervivencia. El pronóstico depende del tamaño y profundidad de la invasión. Conclusiones. El compromiso vesical metastásico es poco frecuente y diagnosticado, puede estar presente en pacientes con melanoma, síntomas irritativos urinarios no específicos y hematuria. Suele ser de mal pronóstico, y requiere de manejo quirúrgico asociado a manejo sistémico.

Palabras clave:

Melanoma; Metástasis de la Neoplasia; Neoplasias de la Vejiga Urinaria; Hematuria; Urología.

\section{ABSTRACT}

Introduction. Metastatic tumor compromise of melanoma to the genitourinary tract is frequent, but metastasis to the bladder is rare, representing less than $2 \%$ of cases. However, autopsies performed on patients with melanoma have found metastases in the bladder in 18-37\% of cases, making it the second incidence after gastric adenocarcinoma. The median survival is usually 6 to 7.5 months. The objective of this work is to present the case of a metastatic melanoma to the bladder, a rare and underdiagnosed condition because most of the time it is asymptomatic. Case Presentation. 62-year-old female patient, with a history of melanoma at the level of the first toe, with surgical and pharmacological management. The reason for consultation was hematuria. Cystoscopy revealed a single solid, erythematous lesion with necrosis and easy bleeding, and a transurethral resection (TUR) was indicated. The pathology found compromise for metastatic ulcerated melanoma. Second-line treatment (Pembrolizumab) was started and presented progression to the upper limbs and relapse at the bladder level. The patient died a year later. Discussion. Melanoma metastases to the genitourinary tract are common, but isolated bladder metastases are rare. Treatment is usually TUR of the lesion, cystectomy, chemotherapy, and radiation therapy. TUR is curative for lesions restricted to the epithelium, although radical cystectomy is usually the therapy of choice in patients with a localized tumor. Pembrolizumab has been shown to increase survival. The prognosis depends on the size and depth of the invasion. Conclusions. Metastatic bladder compromise is rare and underdiagnosed, it may be present in patients with melanoma, nonspecific urinary irritative symptoms, and hematuria. It tends to have a poor prognosis, and requires surgical management associated with systemic management.

Keywords:

Melanoma; Neoplasm Metastasis; Urinary Bladder Neoplasms; Hematuria; Urology.

\section{RESUMO}

Introdução. O comprometimento do tumor metastático do melanoma no trato geniturinário é comum, mas a metástase na bexiga é rara, constituindo menos de $2 \%$ dos casos. Entretanto, em autópsias realizadas em pacientes com melanoma, foi encontrada metástase na bexiga entre $18 \%$ e 37\% dos casos, o que a torna a segunda em incidência após o adenocarcinoma gástrico. A média de sobrevivência é geralmente entre 6 - 7,5 meses. O objetivo deste trabalho é apresentar o caso de um melanoma metastático na bexiga, uma entidade pouco frequente e subdiagnosticada, pois na maioria das vezes é assintomática. Apresentação do caso. Paciente do sexo feminino, 62 anos, com antecedentes de melanoma no nível do hálux, com manejo cirúrgico e farmacológico. Ela consultou por hematúria. A cistoscopia revelou uma única lesão sólida, eritematosa com necrose e sangramento fácil, e foi indicada uma ressecção transuretral (RTU). A patologia mostrou comprometimento de melanoma ulceroso metastático. O tratamento de segunda linha (Pembrolizumab) foi iniciado e a patologia avançou para os membros superiores e uma recaída no nível da bexiga. A paciente morreu um ano depois. Discussão. As metástases de melanoma para o trato geniturinário são frequentes, mas as metástases vesicais isoladas são raras. O tratamento é geralmente RTU da lesão, cistectomia, quimioterapia e radioterapia. A RTU é curativa para lesões restritas ao epitélio, embora a cistectomia radical seja geralmente a terapia de escolha para um paciente com um tumor localizado. O Pembrolizumab demonstrou aumentar a sobrevivência. O prognóstico depende do tamanho e da profundidade da invasão. Conclusões. O comprometimento vesical metastático é raro 
e subdiagnosticado, pode estar presente em pacientes com melanoma, sintomas irritantes urinários não específicos e hematúria. Geralmente tem um prognóstico negativo e requer manejo cirúrgico em associação com manejo sistêmico.

Palavras-chave:

Melanoma; Metástase Neoplásica; Neoplasias da Bexiga Urinária; Hematúria; Urologia.

\section{Introducción}

El melanoma es la presentación más conocida del cáncer de piel. El compromiso tumoral metastásico del melanoma al tracto genitourinario es frecuente, pero la metástasis a vejiga es rara, constituyendo menos del $2 \%$ de los casos $(1,2)$. Sin embargo, en autopsias realizadas a pacientes con melanoma, se han encontrado metástasis en la vejiga en $18-37 \%$ de los casos $(3,4)$, lo que le otorga el segundo lugar en incidencia posterior al adenocarcinoma gástrico (5). El melanoma maligno metastásico vesical se presenta en pacientes entre los 44 a 81 años y no se ha evidenciado prevalencia de género (6).

Las metástasis de melanoma al tracto genitourinario son frecuentes, pero las metástasis vesicales aisladas son raras (7), lo que sugiere que la incidencia es más alta de lo que se cree, lo que se explica porque la mayoría de pacientes son asintomáticos aunque en un $15 \%$ de los casos se presenta hematuria, siendo ésta su forma de presentación más frecuente (8).

El objetivo de este trabajo es presentar el caso de un melanoma metastásico a vejiga, entidad poco frecuente y diagnosticada al ser, la mayoría de las veces, asintomática.

\section{Presentación del caso}

Paciente femenina de 62 años, con antecedente de melanoma a nivel de primer artejo del pie derecho, que requirió desarticulación del mismo en 2011 con posterior progresión a muslo derecho, que requirió vaciamiento linfático inguino-femoral y manejo farmacológico en agosto de 2018 con Pembrolizumab.

Se consultó a urología en septiembre de 2018 por cuadro de hematuria macroscópica de un mes de evolución. La paciente negó tener síntomas irritativos urinarios $\mathrm{u}$ obstructivos así como síntomas constitucionales y no tenía antecedentes toxicológicos de importancia a parte de los reportados por enfermedad de base.

Se tomó ecografía de vías urinarias que reportó una lesión sólida dependiente de la pared vesical derecha de $1.5 \times 1.5 \times 1.2 \mathrm{~cm}$. Se realizó una cistoscopia que evidenció una lesión única redondeada, de aspecto sólido, al nivel de la pared lateral izquierda, de aproximadamente $2 \mathrm{~cm}$, eritematosa, con áreas de necrosis, muy congestiva y de fácil sangrado. Su base de implantación era pequeña y no pediculada.

Se decidió no realizar biopsia con pinza fría porque existía un riesgo importante de hematuria, y, en cambio, se indicó realizar resección transuretral (RTU) de la lesión. El procedimiento se realizó sin complicaciones. En la (Figura 1) se evidencian los hallazgos del RTU.

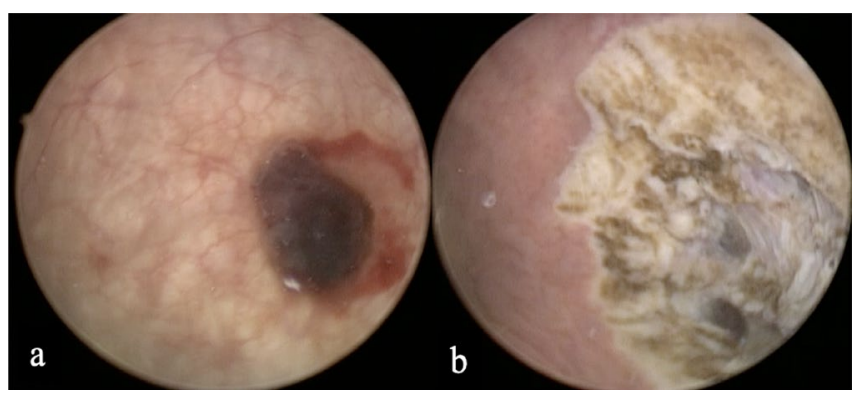

Figura 1. Hallazgos RTU. a) pre resección con lesión única redondeada de aspecto sólido a nivel de pared lateral izquierda, de aproximadamente $2 \mathrm{~cm}$, eritematosa, con áreas de necrosis, muy congestiva, de fácil sangrado, base de implantación pequeña, no pediculada. b) post resección de la lesión.

Fuente: elaborada por los autores.

El reporte de patología demostró hallazgos patológicos de lesión tumoral maligna, constituida por células de núcleos vesiculosos atípicos, con nucléolo prominente y pigmento melánico intracitoplasmático. Lesión que ulcera el urotelio (Figura 2).
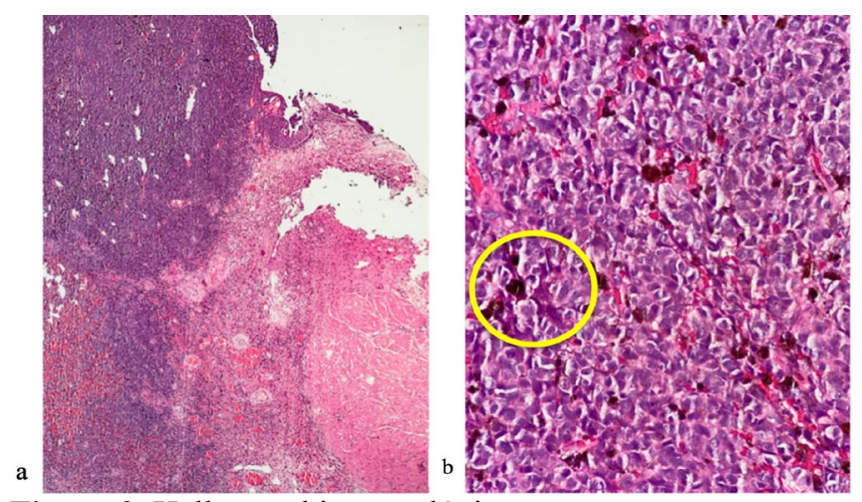

Figura 2. Hallazgos histopatológicos

a) Hematoxilina eosina 4x. Al lado derecho de la foto se observa urotelio y muscular propia, y a la izquierda una lesión tumoral maligna que úlcera el epitelio. b) Hematoxilina eosina 40x. Lesión tumoral está constituida por células de núcleos vesiculosos atípicos con nucleolo prominente y pigmento melánico intracitoplasmático (círculo amarillo).

Fuente: elaborada por los autores. 
Con estos hallazgos se decidió que oncología clínica manejara el caso y esta especialidad decidió iniciar segunda línea con Pembrolizumab. A pesar de este manejo la paciente presentó: progresión de la enfermedad a miembros superiores y recidiva a nivel vesical a los 7 meses post operatorios. No presentó nuevos episodios de hematuria y, finalmente, falleció en agosto de 2019 por compromiso metastásico de la enfermedad.

La paciente falleció extra institucionalmente, por lo que se sostuvo comunicación con su familiar quien confirma la muerte por secuelas de la enfermedad.

\section{Discusión}

El melanoma metastásico a vejiga es una entidad poco frecuente y diagnosticada porque no todos los pacientes presentan síntomas asociados que permitan sospechar el compromiso metastásico a nivel vesical, como en el caso reportado previamente.

Dasgupta y Brasfield encontraron, en las autopsias de 125 pacientes con melanoma, una tasa de $18-37 \%$ de enfermedad metastásica en vejiga $(1,2)$. Así mismo, Ganem y Batal revisaron 80 casos de diseminación metastásica a la vejiga desde focos distantes y encontraron que $22.5 \%$ eran secundarios al melanoma, siendo éste el segundo en incidencia posterior al adenocarcinoma gástrico (3).

Hasta la fecha, existen menos de 20 casos reportados en la literatura de melanoma vesical primario $(6,8)$. Respecto a la sintomatología, se conoce que la mayoría de los pacientes son asintomáticos y sólo $15 \%$ presentan hematuria, su forma de presentación más frecuente $(5,9)$. Por este motivo, a todo paciente con historia previa de melanoma, que desarrolla síndrome miccional (dado por disuria, dificultad para la micción, sensación de tenesmo vesical, polaquiuria o nicturia) y hematuria, se recomienda realizarle estudios endoscópicos para excluir el compromiso metastásico a nivel vesical (811). Estudios en autopsias han demostrado que hasta $18 \%$ de los pacientes con enfermedad metastásica tienen compromiso vesical no conocido (12). No se encontró, dentro de los artículos seleccionados, información o estudios basados en población colombiana $\mathrm{o}$ latinoamericana.

En el momento en que se realice la cistoscopia se puede evidenciar una lesión única, con base de implantación o varias lesiones como pequeños nódulos, pigmentados y elevados que cubren el urotelio, como la encontrada en la paciente $(10,13)$. A nivel histopatológico, se evidencian células melanocíticas atípicas similares al tumor primario. Con sólo la morfología y los pigmentos pardos positivos de Masson-Fontana se puede confirmar el diagnóstico $(1,14)$.

El tratamiento propuesto para estos casos es la resección transuretral de la lesión, cistectomía parcial, cistectomía radical, quimioterapia y radioterapia. La RTU es curativa para las lesiones restringidas al epitelio $(14,15)$, aunque la mayoría de los autores concluyen que la cistectomía radical es la terapia de elección ante un paciente con tumor localizado, ya que este tipo de tumor suele recidivar luego de la escisión local (16).

La PDI (en inglés Programmed Death-ligand 1) es una proteína de control inmune con ligandos PDL1 y PDL2 que interactúan en la membrana de los linfocitos y suprime la actividad de los linfocitos T. Se han desarrollado dos anti cuerpos monoclonales anti PD1 (Nivolumab y Pembrolizumab) que se utilizan como tratamientos de segunda línea, que han demostrado modulación del sistema inmunitario, la cual puede traducirse en respuestas tumorales objetivas de un $30 \%$ de los pacientes y un beneficio a largo plazo en los pacientes respondedores (17). El Pembrolizumab ha demostrado mejorar la supervivencia en pacientes con melanoma metastásico que no han respondido a otros esquemas de tratamiento (17-19).

La elección del tratamiento debe basarse en el pronóstico, estado de salud, la presencia o no de síntomas locales, la profundidad y el tamaño de la lesión. En el presente caso, a parte de la RTU, oncología clínica decidió iniciar manejo de segunda línea con Pembrolizumab, dado que la paciente presentó recaída tumoral estando en manejo con Nivolumab.

El pronóstico depende del tamaño y profundidad de la invasión (14), la media de supervivencia suele ser entre 6 y 7.5 meses y nunca supera los 3 años $(8,20)$. La paciente presentó progresión de su enfermedad a miembros superiores y recidiva a nivel vesical en 7 meses, con muerte a los 12 meses desde que consultó a urología.

\section{Conclusión}

El melanoma metastásico a vejiga es una enfermedad poco frecuente y poco diagnosticada, dado que muy pocos pacientes presentan síntomas asociados. Siempre debe sospecharse en pacientes con antecedente de melanoma y síntomas irritativos urinarios no específicos y hematuria. Por este motivo se recomienda realizar una cistoscopia en todos los pacientes sintomáticos y 
con el antecedente. El melanoma metastásico a vejiga suele ser de mal pronóstico y, por lo general, requiere de manejo quirúrgico con RTU (como el caso reportado) o cistectomía, asociado a manejo sistémico.

El melanoma primario del aparato genitourinario es extremadamente raro y representa el $0.2 \%$ de todos los melanomas. Se han reportado en literatura menos de 20 casos de melanoma primario en vejiga hasta el 2013. Su diagnóstico se debe confirmar con inmunohistoquímica, y su tratamiento consiste en la cistectomía radical. Sin embargo, presenta un mal pronóstico, encontrándose una mortalidad alta dentro de los primeros 3 años.

\section{Conflictos de intereses}

Los autores declaran no tener conflictos de intereses.

\section{Financiación}

Para la realización de este estudio, no existió ningún tipo de financiación externa a los autores.

\section{Consideraciones éticas}

Para la publicación de este artículo se contó con la autorización del paciente por medio del consentimiento informado en donde autoriza la manipulación de los datos con fines académicos investigativos según la resolución 8430 de 1993.

\section{Referencias}

1. Nualyong C, Woranisarakul V, Tantranont N, Chotikawanich E, Shrestha S, Taweemonkongsap T. Metastatic Malignant Melanoma of the Urinary Bladder: A Case Report and Review of the Literature. Siriraj Med J [Internet]. 2018;70(3):254-259. Recuperado a partir de: https:/he02.tci-thaijo.org/ index.php/sirirajmedj/article/view/129734

2. Bates AW, Baithun SI. Secondary neoplasms of the bladder are histological mimics of nontransitional cell primary tumours: clinicopathological and histological features of 282 cases. Histopathology [Internet]. 2000;36(1):32-40. doi: https://doi.org/10.1046/ j.1365-2559.2000.00797.x

3. Ganem E, Batal J. Secondary Malignant Tumors of the Urinary Bladder Metastatic from Primary Foci in Distant Organs. J Urol [Internet]. 1956;75(6):965-72. doi: https://doi.org/10.1016/s0022-5347(17)66911-8
4. Das-Gupta T, Brasfield R. Metastatic melanoma: A clinicopathological study. Cancer [Internet]. 1964;17(10):1323-39. doi: https:/doi. org/10.1002/1097-0142(196410)17:10\%3C1323::aidcncr2820171015\%3E3.0.co;2-n

5. Klinger ME. Secondary Tumors of the GenitoUrinary Tract. J Urol [Internet]. 1951;65(1):144-53. doi : https://doi.org/10.1016/s0022-5347(17)68470-2

6. Niederberger CS, Lome LG. Primary malignant melanoma of urinary bladder. Urology [Internet]. 1993;41(1):72-4. doi: https://doi.org/10.1016/00904295(93)90250-e

7. Efesoy O, Cayan S. Bladder metastasis of malignant melanoma: a case report and review of literature. Med Oncol [Internet]. 2011;28(1):667-9. doi: https://doi. org/10.1007/s12032-010-9730-x

8. Añón-Requena MJ, Muñoz-Arias G, RamírezChamorro R. Melanoma maligno metastásico en vejiga urinaria. Actas Urol Esp [Internet]. 2015;39(10):6523. doi: https://doi.org/10.1016/j.acuro.2015.05.009

9. Demirkesen O, Yaycioglu O, Uygun N, Demir G, Yalcin V. A Case of Metastatic Malignant Melanoma Presenting with Hematuria. Urol Int [Internet]. 2000;64(2):118-20. doi: https://doi. org $/ 10.1159 / 000030506$

10. Casimiro-Guzmán L, Hernández-Román LU, CruzContreras LH, Chávez-Martínez S. Melanoma en vejiga, reporte de un caso y revisión de la literatura. Rev Mex de Urol [Internet]. 2015;75(5):306-9. doi: https://doi.org/10.1016/j.uromx.2015.08.001

11. Moore NA, Stunell HJ, Sizer B, Casey RG. Malignant melanoma metastasis to the urinary bladder: a rare cystoscopic finding. J Clin Urol [Internet]. 2015;8(2):143-6. doi: https://doi. org/10.1177\%2F2051415813510712

12. Wisenbaugh ES, Stanton ML, Grimsby GM, Tyson MD, Castle EP. Metastatic Malignant Melanoma to the Bladder: A Case Series. Current Urology [Internet]. 2012;6(1):53-6. doi: https://doi. org/10.1159/000338872

13. Meunier R, Pareek G, Amin A. Metastasis of Malignant Melanoma to Urinary Bladder: A Case Report and Review of the Literature. Case Rep Pathol [Internet]. 2015; 2015:1-6. doi: https://dx.doi. org $/ 10.1155 \% 2 \mathrm{~F} 2015 \% 2 \mathrm{~F} 173870$

14. Venyo AK-G. Melanoma of the Urinary Bladder: A Review of the Literature. Surg Res Pract [Internet]. 2014;2014:1-13. doi: https://dx.doi. org $10.1155 \% 2 \mathrm{~F} 2014 \% 2 \mathrm{~F} 605802$

15. Khan M, O'Kane D, Plessis JD, Hoag N, Lawrentschuk N. Primary malignant melanoma of the urinary bladder and ureter. Can J Urol [Internet]. 2016;23(1):8171-5. Recuperado a partir de: https:// pubmed.ncbi.nlm.nih.gov/26892061/ 
16. Lamichhane N, Dhakal HP. Melanoma of Urinary Bladder Presented as Acute Urine Retention. J Urol Res [Internet]. 2016;3(3):1054. Recuperado a partir de: https://www.researchgate.net/ publication $/ 303451018$ Melanoma of Urinary Bladder Presented as Acute Urine Retention

17. Specenier P. Pembrolizumab use for the treatment of advanced melanoma. Expert Opin Biol Ther [Internet]. 2017;17(6):765-80. doi: https://doi.org/10 $.1080 / 14712598.2017 .1309388$

18. Deeks ED. Pembrolizumab: A Review in Advanced Melanoma. Drugs [Internet]. 2016;76:375-86. doi: https://doi.org/10.1007/s40265-016-0543-x
19. Lee CSD, Komenaka IK, Hurst-Wicker KS, Deraffele G, Mitcham J, Kaufman HL. Management of metastatic malignant melanoma of the bladder. Urology [Internet]. 2003;62(2):351. doi: https://doi. org/10.1016/s0090-4295(03)00354-6

20. Crosby T, Fish R, Coles B, Mason M. Systemic treatments for metastatic cutaneous melanoma. Cochrane Database Syst Rev [Internet]. 2018;2(2). doi: $\quad$ https://dx.doi.org/10.1002\%2F14651858. CD001215.pub2 